\title{
MYDGF attenuates podocyte injury and proteinuria by activating Akt/BAD signal pathway in mice with diabetic kidney disease
}

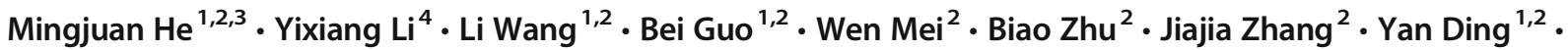 \\ Biying Meng ${ }^{1,2} \cdot$ Liming Zhang ${ }^{1,2} \cdot$ Lin Xiang $^{2} \cdot$ Jing Dong ${ }^{2} \cdot$ Min Liu $^{2} \cdot$ Lingwei Xiang $^{5} \cdot$ Guangda Xiang $^{1,2}$
}

Received: 23 December 2019 / Accepted: 27 April 2020 / Published online: 25 June 2020

(C) Springer-Verlag GmbH Germany, part of Springer Nature 2020

\begin{abstract}
Aims/hypothesis Myeloid-derived growth factor (MYDGF), mainly secreted by bone marrow-derived cells, has been known to promote glucagon-like peptide-1 production and improve glucose/lipid metabolism in mouse models of diabetes, but little is known about the functions of MYDGF in diabetic kidney disease (DKD). Here, we investigated whether MYDGF can prevent the progression of DKD.

Methods In vivo experiments, both loss- and gain-of-function strategies were used to evaluate the effect of MYDGF on albuminuria and pathological glomerular lesions. We used streptozotocin-treated $M y d g f$ knockout and wild-type mice on high fat diets to induce a model of DKD. Then, albuminuria, glomerular lesions and podocyte injury were evaluated in $M y d g f$ knockout and wild-type DKD mice treated with adeno-associated virus-mediated $M y d g f$ gene transfer. In vitro and ex vivo experiments, the expression of slit diaphragm protein nephrin and podocyte apoptosis were evaluated in conditionally immortalised mouse podocytes and isolated glomeruli from non-diabetic wild-type mice treated with recombinant MYDGF.

Results MYDGF deficiency caused more severe podocyte injury in DKD mice, including the disruption of slit diaphragm proteins (nephrin and podocin) and an increase in desmin expression and podocyte apoptosis, and subsequently caused more severe glomerular injury and increased albuminuria by $39.6 \%$ compared with those of wild-type DKD mice $(p<0.01)$. Inversely, MYDGF replenishment attenuated podocyte and glomerular injury in both wild-type and Mydgf knockout DKD mice and then decreased albuminuria by $36.7 \%$ in wild-type DKD mice $(p<0.01)$ and $34.9 \%$ in Mydgf knockout DKD mice $(p<0.01)$. Moreover, recombinant MYDGF preserved nephrin expression and inhibited podocyte apoptosis in vitro and ex vivo. Mechanistically, the renoprotection of MYDGF was attributed to the activation of the Akt/Bcl-2-associated death promoter (BAD) pathway.

Conclusions/interpretation The study demonstrates that MYDGF protects podocytes from injury and prevents the progression of DKD, providing a novel strategy for the treatment of DKD.
\end{abstract}

Keywords Diabetic kidney disease $\cdot$ Myeloid-derived growth factor $\cdot$ Podocyte injury

\section{Abbreviations \\ AAV Adeno-associated virus \\ BAD Bcl-2-associated death promoter}

Electronic supplementary material The online version of this article (https://doi.org/10.1007/s00125-020-05197-2) contains peer-reviewed but unedited supplementary material, which is available to authorised users.

Guangda Xiang

Guangda64@hotmail.com

Lingwei Xiang

Ynp8@cdc.gov

Extended author information available on the last page of the article

$\begin{array}{ll}\text { Bcl-2 } & \text { B-cell lymphoma 2 } \\ \text { BMDC } & \text { Bone marrow-derived cell } \\ \text { BMSC } & \text { Bone marrow mesenchymal stem cell } \\ \text { BMMNC } & \text { Bone marrow mononuclear cell } \\ \text { BMC } & \text { Bone marrow cell } \\ \text { BUN } & \text { Blood urea nitrogen } \\ \text { DKD } & \text { Diabetic kidney disease } \\ \text { GBM } & \text { Glomerular basement membrane } \\ \text { GLP-1 } & \text { Glucagon-like peptide-1 } \\ \text { HFD } & \text { High-fat diet } \\ \text { HG } & \text { High glucose } \\ \text { KO } & \text { Knockout } \\ \text { MA } & \text { Mannitol }\end{array}$




\section{Research in context}

\section{What is already known about this subject?}

- Myeloid-derived growth factor (MYDGF) is a paracrine-acting protein produced by bone marrow-derived monocytes and macrophages

- A previous study has indicated that MYDGF promotes glucagon-like peptide-1 production and improves glucose/lipid metabolism in a mouse model of diabetes

\section{What is the key question?}

- Does MYDGF affect the progression of diabetic kidney disease?

\section{What are the new findings?}

- MYDGF ameliorates albuminuria and glomerular injury in a mouse model of diabetic kidney disease

- MYDGF preserves nephrin expression and protects against podocyte apoptosis both in vivo and in vitro

- The molecular mechanisms underlying these beneficial effects of MYDGF involve the activation of the Akt/BAD signalling pathway

\section{How might this impact on clinical practice in the foreseeable future?}

- The study suggests a critical role for MYDGF in preventing the development of diabetic kidney disease by protecting podocytes from injury and improving podocyte function through the activation of the Akt/BAD pathway, opening up a potential new therapeutic strategy for treating diabetic kidney disease

$\begin{array}{ll}\text { MYDGF } & \text { Myeloid-derived growth factor } \\ \text { NG } & \text { Normal glucose } \\ \text { PAS } & \text { Periodic acid-Schiff } \\ \text { PKA } & \text { Protein kinase A } \\ \text { rMYDGF } & \text { Recombinant MYDGF } \\ \text { RSK } & \text { p90 ribosomal S6 kinase } \\ \text { siRNA } & \text { Small interfering RNA } \\ \text { STZ } & \text { Streptozotocin } \\ \text { S6K } & \text { p70 ribosomal S6 kinase } \\ \text { TEM } & \text { Transmission electron microscopy } \\ \text { UACR } & \text { Urinary albumin/creatinine ratio } \\ \text { WT } & \text { Wild-type } \\ \text { WT-1 } & \text { Wilms' tumour 1 }\end{array}$

\section{Introduction}

Diabetic kidney disease is one of the most common complications of diabetes and has become the leading cause of endstage renal disease [1]. As the earliest appearing symptom, proteinuria is the hallmark of diabetic kidney disease (DKD) and is caused by disruption to the glomerular filtration barrier [2]. Podocytes present on the outer surface of the glomerular basement membrane (GBM), which contributes to maintaining the structure and function of the glomerular filtration barrier. Podocyte injury, such as alterations in the structures of podocytes or loss of podocytes, affects the integrity of the filtration barrier, resulting in proteinuria and glomerulosclerosis [3, 4]. Moreover, apoptosis is one of the main reasons for podocyte loss, since podocytes are highly specialised, terminally differentiated and unable to be replaced or regenerated [5-7]. Therefore, protecting podocytes from injury is a promising therapeutic target for DKD treatment.

Bone marrow-derived cells (BMDCs) are a pool of progenitor and pluripotent stem cells including endothelial progenitor cells, haematopoietic stem cells and mesenchymal stromal cells that secrete a variety of cytokines, growth factors, exosomes and microvesicles [8]. Evidence in recent years has shown that BMDCs, such as bone marrow mesenchymal stem cells (BMSCs) and bone marrow mononuclear cells (BMMNCs), inhibit the progression of DKD $[9,10]$. In rodent models, intravenous injection of BMSCs can preserve renal structure and ameliorate the development of diabetic nephropathy [11], and BMMNC treatment also improves glomerular function in early diabetic nephropathy [12]. In clinical trials, allogeneic BMSC transplantation can improve the glomerular filtration rate [13], and autologous BMMNC infusion also ameliorates albuminuria in diabetic nephropathy patients [14]. Moreover, increasing numbers of studies have demonstrated that the paracrine effect, as an alternative mechanism by which BMDCs secrete biologically active molecules, is a promising strategy for the treatment of DKD $[8,15,16]$. Myeloid-derived growth factor (MYDGF, also known as C19orf10) is also a paracrine-acting protein produced by bone marrow-derived monocytes and macrophages [17]. Our previous study has found that MYDGF can promote intestinal glucagon-like peptide-1 (GLP-1) production and improve glucose/lipid metabolism in a mouse model of type 2 diabetes 
[18]. However, whether MYDGF also participates in the development of DKD remains unknown. Therefore, in this study, we investigated the effects of MYDGF on DKD and explored the possible mechanisms involved.

\section{Methods}

\section{Human study}

A total of 60 male type 2 diabetic patients with DKD admitted to the Department of Endocrinology, General Hospital of Central Theater Command from October 2018 to May 2019 were enrolled in this study. During the same period, 20 agematched healthy male participants were selected as a control group. All the participants enrolled in this study signed informed consent. The study was approved by the ethics committee of the General Hospital of Central Theater Command. The eligibility criteria are provided in electronic supplementary material (ESM) Methods.

Clinical examination BMI and blood pressure were measured. See ESM Methods for further details.

Blood biochemical analysis $\mathrm{HbA}_{1 \mathrm{c}}$, serum total cholesterol, triacylglycerol, HDL-cholesterol, LDL-cholesterol, creatinine and blood urea nitrogen (BUN) were measured, and eGFR was estimated. Plasma MYDGF level was tested in duplicate. See ESM Methods.

Urine biochemical analysis All participants underwent a $24 \mathrm{~h}$ urinary albumin excretion test. See ESM Methods.

\section{Animals and treatments}

Mydgf knockout mice Mydgf knockout (KO) and wild-type (WT) littermate mice were produced by mating $M y d g f^{+/-}$ breeders of C57BL/6J background purchased from Shanghai Southern Model Biotechnology Company, China.

DKD mice Streptozotocin (STZ; Sigma-Aldrich, St Louis, MO, USA)-treated mice fed with high-fat diet (HFD) (41\% energy from fat, $42 \%$ carbohydrate and $17 \%$ protein) were used to produce DKD models following previous studies $[19,20]$. The WT and KO male mice aged 4 weeks were both fed an HFD for 4 weeks and then i.p. injected with STZ $(50 \mathrm{mg} / \mathrm{kg}$ per day) for 5 consecutive days to generate a mouse model of type 2 diabetes [18]. Nine days later, the mice were considered diabetic when their nonfasting blood glucose levels exceeded $13.9 \mathrm{mmol} / \mathrm{l}$ for 2 consecutive days [21]. After the diabetic model was produced, the mice were maintained for an additional 6 weeks of continued HFD feeding.
Adeno-associated-virus-treated mice The $M y d g f$ (GenBank accession number NM_080837.2) cDNA was synthesised and adeno-associated virus (AAV)-MYDGF was constructed as previously $[18,22]$. The mice received a single injection of AAV-MYDGF or AAV-GFP at a dose of $1 \times 10^{12}$ viral genomes through the tail vein. For small interfering RNA (siRNA) treatment, the $A k t$-specific siRNA (GenBank accession number NM 009652.3) and the scrambled control siRNA oligonucleotides were obtained and AAV-siRNA/Akt (siAkt) and AAV-siRNA/control (siCON) were produced as previously $[18,23]$. Both kidneys of the mice were treated with siAkt or siCON at a dose of $1.6 \times 10^{11}$ viral genomes using orthotopic injection [24, 25].

Bone marrow chimeric mice Bone marrow cells (BMCs) were flushed from the femurs and tibias of the WT and KO mice and transplanted into lethally irradiated $(7.5 \mathrm{~Gy})$ recipient $\mathrm{WT}$ and $\mathrm{KO}$ mice through the tail vein $\left(1 \times 10^{6}\right.$ cells per mouse $)$ as previously described [17].

All the experiments followed the National Institutes of Health Guidelines for the Use of Laboratory Animals. All the animal procedures were approved by the animal ethics committee of the General Hospital of Central Theater Command. See ESM Methods.

\section{Metabolic and biochemical analyses}

Glucose and insulin tolerance tests, blood pressure, blood biochemical analysis and urine biochemical analysis are detailed in ESM Methods.

\section{Histology}

For the morphometric assessment, kidney sections were stained with a periodic acid-Schiff (PAS) kit (SigmaAldrich). For immunofluorescence staining, MYDGF, nephrin and podocin antibodies were used. To identify podocyte apoptosis, Wilms' tumour 1 (WT-1) and TUNEL double staining and nephrin and cleaved caspase-3 double staining were performed. The analyses are detailed in ESM Methods. The antibodies are detailed in ESM Table 1.

\section{Transmission electron microscopy}

Several $1 \mathrm{~mm}$ cubes from the renal cortex were cut, fixed, stained, embedded for transmission electron microscopy (TEM) and quantified, as described previously [26, 27]. See ESM Methods.

\section{Glomeruli isolation and culture}

Briefly, mice were perfused with Dynabeads M-450 (Invitrogen, Carlsbad, CA, USA). After perfusion, the kidney 
tissue was digested, filtered and transferred to a magnetic particle concentrator, which gathered the glomeruli that contained Dynabeads. After isolation, the glomeruli were incubated in DMEM under various experimental conditions. See ESM Methods.

\section{Cell culture and treatments}

Conditionally immortalised mouse podocytes (MPC5) were purchased from the Cell Bank of the BeNa Culture Collection of Beijing (BNCC337685) and cultured in RPMI1640 medium (Gibco, Grand Island, NY, USA) containing 10\% FBS (Invitrogen) and $5.5 \mathrm{mmol} / \mathrm{l}$ glucose. Podocytes were authenticated by morphology examination and nephrin expression. No mycoplasma contamination was detected.

\section{Apoptosis assays}

Apoptosis was determined using caspase-3 activity (detailed in ESM Methods) and flow cytometry (Beckman-Coulter, Indianapolis, IN, USA) after double staining with annexin VFITC and propidium iodide (eBioscience, San Diego, CA, USA).

\section{RT-PCR and western blot}

RT-PCR was performed as previously [28]. The primer sequences are listed in ESM Table 2. Western blot was performed on isolated glomeruli or cell extracts as described previously [29]. The antibodies are listed in ESM Table 1.

\section{Statistical analysis}

All the quantified data are expressed as the means \pm SEM. Data between groups were compared by the unpaired Student's $t$ test or one-way ANOVA followed by Tukey's post hoc test for multiple comparisons. A $p$ value $<0.05$ was considered significant.

\section{Results}

\section{MYDGF levels decrease in human participants and mice with DKD}

Our previous report found that the plasma levels of MYDGF decreased in diabetic patients and mice [18]. Thus, we further explored MYDGF expression in DKD. Sixty male participants with type 2 diabetes and 20 age-matched healthy male control participants were enrolled for measurement of their plasma MYDGF levels (participant characteristics are detailed in ESM Table 3). According to the degree of albuminuria, patients were classified into a normoalbuminuric group, a microalbuminuric group and a macroalbuminuric group
Fig. 1 MYDGF expression declines in patients and mice with DKD, and MYDGF deficiency accelerates albuminuria and glomerular injury in DKD mice. (a) MYDGF levels in normoalbuminuric, microalbuminuric and macroalbuminuric diabetic and healthy participants $(n=20$ per group). (b, c) Plasma MYDGF content (b) and bone marrow Mydgf mRNA expression (c) in DKD mice and control mice $(n=10$ per group). The mRNA expression of the DKD mice is relative to that of the control mice. (d) Experiment schedule of the HFD/STZ-induced DKD mouse model study in Mydgf KO and WT mice $(n=8$ mice per group). (e) Urinary albumin levels were measured at the termination of the study ( $n=6$ mice per group). (f) Representative PAS staining and (g, h) quantitative analyses of mesangial matrix expansion and glomerular volume in glomeruli ( $n=4$ mice per group). Scale bar, $20 \mu \mathrm{m}$; original magnification $\times 400$. (i) Western blots and quantification analyses of fibronectin in glomerular lysates ( $n=3$ mice per group). (j-l) The mRNA levels of fibronectin, $T g f-\beta$ (also known as $T g f b 1$ ) and collagen IV in glomerular lysates were determined using RT-PCR $(n=6$ mice per group). The mRNA expression of the mice is relative to that of the WTCON mice. Data are shown as the mean \pm SEM. Student's $t$ test was employed for comparisons between two groups. One-way ANOVA followed by Tukey's post hoc test for multiple comparisons was used for groups of three or more. $* p<0.05, * * p<0.01$ vs WT-CON group (except as otherwise shown in a); ${ }^{\dagger \dagger} p<0.01$ vs KO-CON group; ${ }^{\ddagger} p<0.05,{ }^{\sharp} p<0.01$ vs WT-DKD group. CON, control; FN, fibronectin; Macro., macroalbuminuric; Micro., microalbuminuric; MYDGF, myeloid-derived growth factor; Normo., normoalbuminuric; Vol., volume

(ESM Table 3). The results showed that the plasma MYDGF levels in diabetic participants were lower than those of control participants (Fig. 1a). Among the three diabetic groups, the plasma MYDGF levels decreased in the microalbuminuric group and further declined in the macroalbuminuric group compared with the normoalbuminuric group (Fig. 1a). For the mice, the plasma MYDGF levels and Mydgf expression in bone marrow decreased in HFD/STZ-induced DKD mice compared with control mice (Fig. 1b, c). These results indicate that MYDGF may be associated with the development of DKD.

\section{MYDGF deficiency aggravates albuminuria, glomerular injury and metabolic characteristics in DKD mice}

As mentioned above, some relationships were found between plasma MYDGF and DKD, and these results encouraged us to explore further the effect of MYDGF deficiency on DKD. We first generated Mydgf global KO mice, and MYDGF expression in the plasma and bone marrow were tested, which confirmed the absence of MYDGF in the KO mice (ESM Fig. 1a, b).

HFD/STZ-induced DKD models in WT and KO mice were then generated (Fig. 1d). In the DKD groups, the urinary albumin/creatinine ratio (UACR) levels increased, being $39.6 \%$ higher in the KO-DKD mice than the WT-DKD mice $(p<0.01)$ (Fig. 1e), with no significant changes in BUN or serum creatinine between the two groups (ESM Table 4). Moreover, PAS staining showed that the glomerular volume 

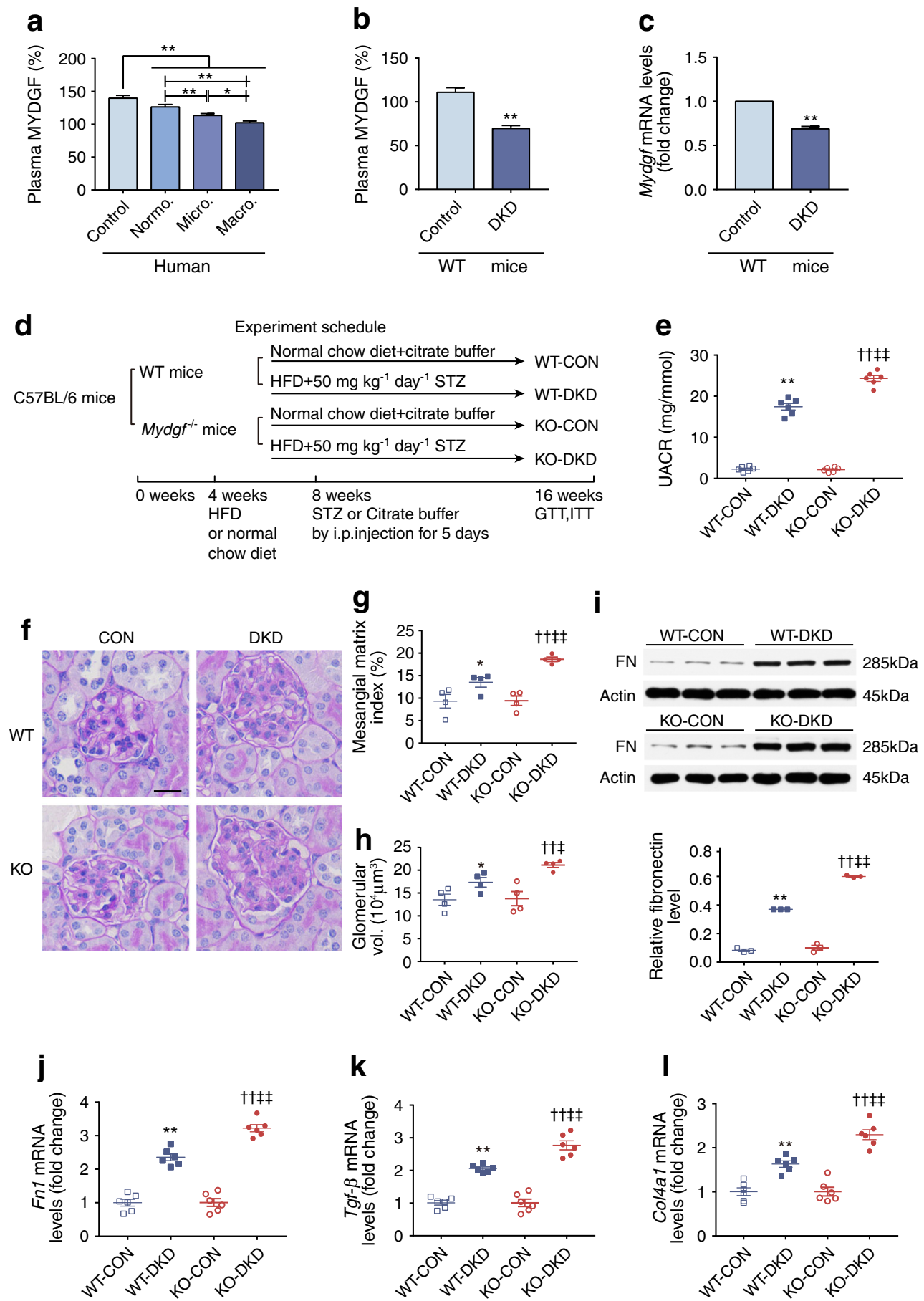

and mesangium expansion increased in the KO-DKD mice compared with the WT-DKD mice (Fig. 1f-h). We also examined the glomerular expression for profibrotic molecules (fibronectin, TGF- $\beta$ and collagen IV). Western blot and RTPCR analyses showed that the levels of the profibrotic molecules were elevated in the KO-DKD mice compared with the WT-DKD mice (Fig. 1i-1). These results illustrate that loss of MYDGF may accelerate albuminuria, glomerulomegaly and glomerulosclerosis in DKD.
Considering that metabolic disturbance is the primary characteristic of DKD [30], we further investigated metabolic indicators. First, the DKD mice developed glucose intolerance and insulin resistance, and the KO-DKD mice displayed more severe impairment in glucose tolerance and insulin sensitivity than the WT-DKD mice (ESM Fig. 2a-d). Second, the DKD groups had dyslipidaemia, and lack of MYDGF resulted in a worse lipid profile (ESM Table 4). Third, MYDGF deficiency led to greater weight gain in KO-DKD mice than in WT-DKD 
Fig. 2 MYDGF deficiency exacerbates podocyte injury and apoptosis in DKD mice. (a) Representative

immunofluorescence images of nephrin in glomeruli. Scale bar, $20 \mu \mathrm{m}$; original magnification $\times 400$. (b) Representative immunofluorescence images of podocin in glomeruli. Scale bar, $20 \mu \mathrm{m}$; original magnification $\times 400$. (c) Representative TEM images of glomeruli. Scale bar, $1 \mu \mathrm{m}$; original magnification $\times 5000$. (d) Representative images showing WT-1 (green) and TUNEL (red) double staining. Scale bar, $20 \mu \mathrm{m}$; original magnification $\times 400$. $(\mathbf{e}, \mathbf{f})$ Quantification analyses of mean GBM thickness and mean foot process width in (c) $(n=5$ mice per group). (g) Absolute count of the numbers of glomerular cells positive for both WT-1 and TUNEL (white arrows) as the means of 20 glomeruli $(n=4$ mice per group). (h, i) Western blots and quantification analyses of nephrin and desmin in glomerular lysates ( $n=3$ mice per group). Data are shown as the mean \pm SEM. One-way ANOVA

followed by Tukey's post hoc test for multiple comparisons was used for groups of three or more. $* p<0.05, * * p<0.01$ vs WTCON group; ${ }^{\dagger} p<0.05,{ }^{\dagger \dagger} p<0.01$ vs KO-CON group; ${ }^{* 1} p<0.01$ vs WT-DKD group. CON, control
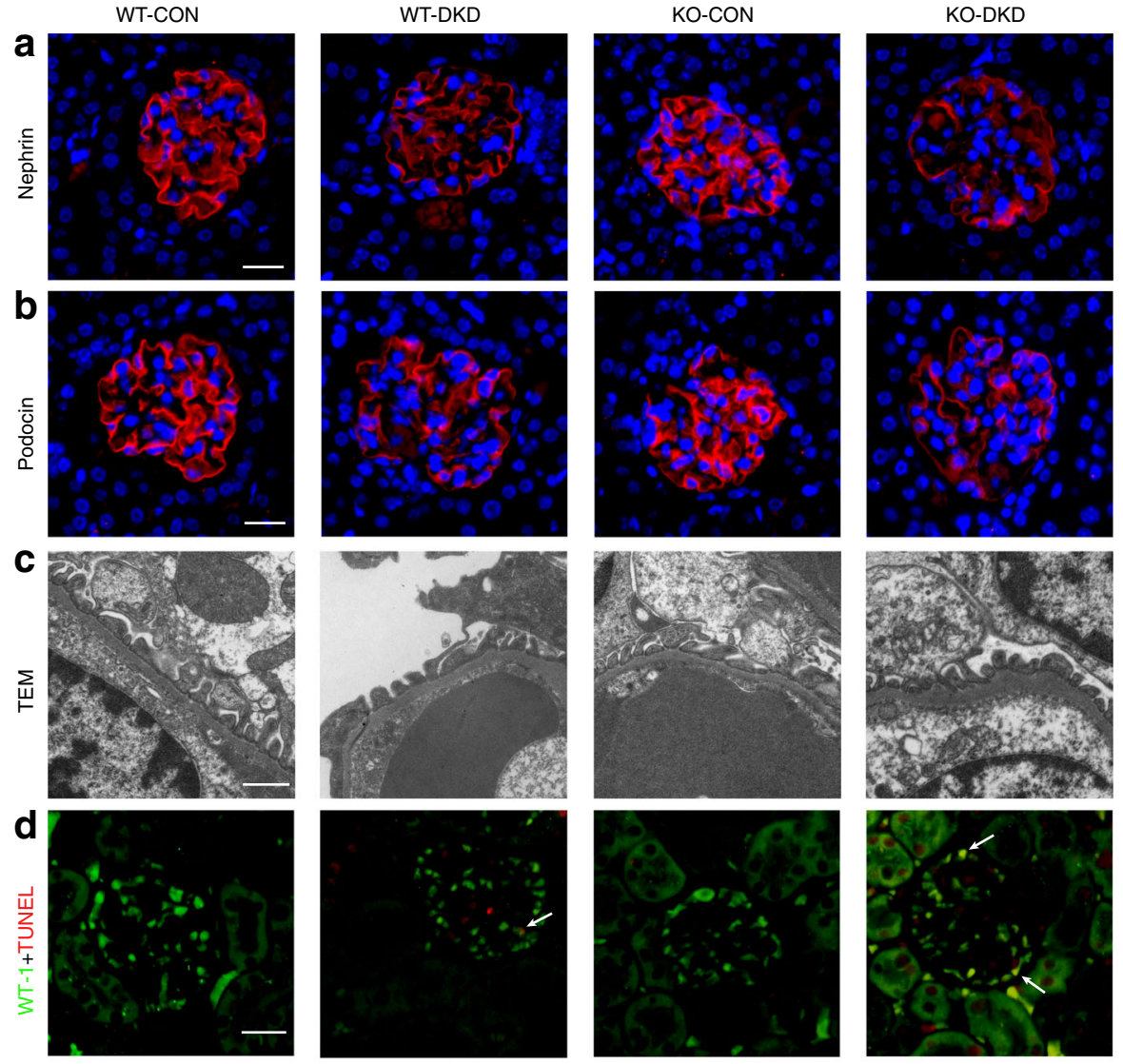

e

f

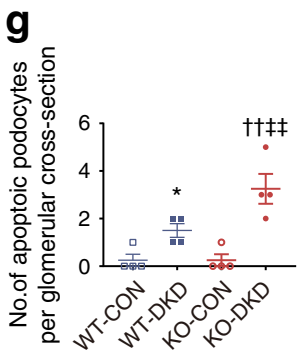

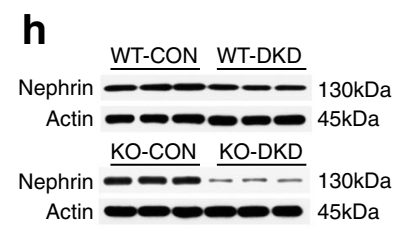

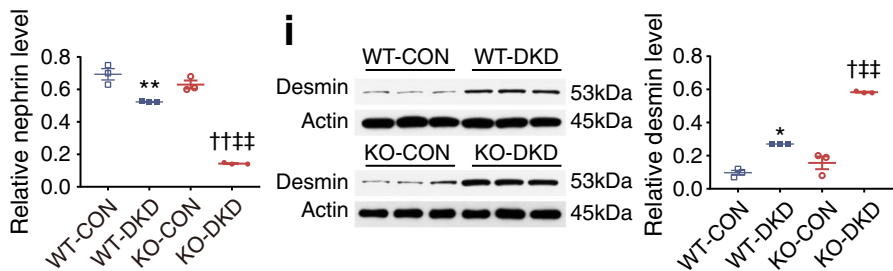

mice, without a difference in food intake between the two groups (ESM Table 4). These data show that loss of MYDGF may aggravate glucose and lipid metabolism in DKD.

\section{MYDGF deficiency promotes podocyte injury in DKD mice}

In view of the important role of podocytes on DKD [31], we next examined the effect of MYDGF deficiency on podocytes.
The results showed that nephrin and podocin expression in the glomeruli decreased in the DKD mice, and the expression further declined in KO-DKD mice compared with WT-DKD mice (Fig. 2a, b). Furthermore, TEM showed podocyte injuries in the DKD groups, as evidenced by GBM thickening and degradation or fusion of the podocyte foot process, and the injuries were aggravated in KO-DKD mice compared with WT-DKD mice (Fig. 2c, e, f). TUNEL staining in WT-1positive cells revealed that the apoptotic podocytes increased in the DKD groups compared with the control groups, and 
loss of MYDGF accelerated podocyte apoptosis in DKD mice (Fig. 2d, g), which was reconfirmed by cleaved caspase-3 staining in nephrin-positive cells (ESM Fig. 3). Western blot analyses showed that MYDGF deficiency decreased nephrin expression and increased desmin expression in DKD mice (Fig. 2h, i). Taken together, these results show that loss of MYDGF may exacerbate podocyte injury in DKD.

\section{MYDGF restoration ameliorates albuminuria, glomerular injury and metabolic characteristics in vivo}

Our animal study showed that MYDGF deficiency aggravated podocyte injury and promoted albuminuria in DKD mice. Thus, we further explored whether MYDGF restoration had a favourable impact on DKD. We first constructed an AAV vector to overexpress MYDGF (ESM Fig. 4a). For AAV treatment, the WT and KO mice received a single injection of AAV-MYDGF or AAV-GFP via the tail vein (Fig. 3a). Six weeks later, the AAV-MYDGF mice had higher plasma MYDGF and liver MYDGF expression than the AAV-GFP mice (ESM Fig. 4be). At the end of the six week period, MYDGF replenishment deceased albuminuria by $36.7 \%$ in the WT mice $(p<0.01)$ and $34.9 \%$ in the KO mice $(p<0.01)$ (Fig. 3b). PAS staining showed that the glomerular volume and mesangium expansion decreased in the mice injected with AAV-MYDGF compared with the mice injected with AAV-GFP (Fig. 3c-e). Consistently, western blot and RT-PCR analyses showed that the profibrotic molecule expression in the glomeruli declined in the WT-MYDGF and KO-MYDGF mice, compared with the respective AAV-GFP mice (Fig. 3f-i). Taken together, these results confirm that MYDGF ameliorates albuminuria, glomerulomegaly and glomerulosclerosis in DKD.

Accordingly, we assessed whether MYDGF restoration affected metabolism. After the 6 week intervention, MYDGF treatment improved hyperglycaemia and dyslipidaemia and decreased body weight gain (ESM Fig. 5e-k, ESM Table 5). These data demonstrate that MYDGF ameliorates glucose and lipid metabolism in DKD.

\section{MYDGF restoration prevents podocyte injury in vivo}

Next, we explored whether MYDGF restoration has a positive effect on podocytes in DKD mice. The results showed that nephrin and podocin expression was preserved in the glomeruli of both WT and KO mice injected with AAV-MYDGF compared with mice injected with AAV-GFP (Fig. 4a, b). TEM also showed that podocyte injuries were attenuated in both WT-MYDGF and KO-MYDGF mice compared with their respective AAV-GFP groups (Fig. 4c, e, f). TUNEL staining revealed that podocyte apoptosis decreased in the WT-MYDGF and KO-MYDGF mice compared with the WT-GFP and KO-GFP mice (Fig. 4d, g), reconfirmed by cleaved caspase-3 staining (ESM Fig. 6), which indicated that MYDGF inhibited podocyte apoptosis. Additionally, western blot analyses showed that MYDGF treatment increased nephrin expression and decreased desmin expression in DKD mice (Fig. 4h). These results illustrate that MYDGF attenuates podocyte injury in DKD.

\section{MYDGF prevents podocyte injury in vitro and ex vivo}

To further corroborate the direct benefits of MYDGF on podocytes, we used podocytes from non-diabetic WT mice as an in vitro system. Podocytes pretreated with $25 \mathrm{mmol} / \mathrm{l}$ glucose (high glucose [HG]) for $24 \mathrm{~h}$ significantly induced cell apoptosis, which was chosen in the following study (ESM Fig. 7a, b). Additionally, exposure of the HG-induced podocytes to recombinant MYDGF (rMYDGF) prevented cell apoptosis in a concentration- and time-dependent manner (ESM Fig. 7c, d). Accordingly, rMYDGF treatment at $100 \mathrm{ng} / \mathrm{ml}$ for $24 \mathrm{~h}$ was used subsequently.

As shown in Fig. 5a, b, nephrin expression decreased under HG conditions, and rMYDGF treatment attenuated this effect. Moreover, flow cytometry analysis showed that more apoptotic podocytes were observed in the HG group than in the normal glucose (NG) group or the mannitol (MA) group, and rMYDGF treatment remitted this effect (Fig. 5e, f). Consistently, the expression of the antiapoptotic protein Bcell lymphoma 2 (Bcl-2) increased and the expression of the proapoptotic Bax and cleaved caspase-3 decreased in the HGinduced podocytes treated with rMYDGF (Fig. 5a, c, d).

In addition, glomeruli isolated from non-diabetic WT mice were used as an ex vivo system. Pretreatment of mouse glomeruli with $25 \mathrm{mmol} / \mathrm{l} \mathrm{HG}$ for $96 \mathrm{~h}$ showed impaired nephrin expression and induced cell apoptosis, which was used subsequently (ESM Fig. 8a, b). Based on the rMYDGF treatment in vitro, the HG-induced glomeruli were subsequently treated with or without $100 \mathrm{ng} / \mathrm{ml} \mathrm{rMYDGF}$ for $24 \mathrm{~h}$. In parallel, the nephrin expression in glomeruli significantly decreased under HG conditions, and rMYDGF abated the effect (Fig. 5g, h). Consistently, rMYDGF treatment increased Bcl-2 expression and decreased the Bax and cleaved caspase-3 expression in HG groups (Fig. 5g, i, j). Taken together, these data further confirm that MYDGF attenuates HG-induced podocyte injury.

\section{Akt/BAD signal is required for the benefits of MYDGF in DKD mice}

We next explored the possible mechanisms through which MYDGF exerts protective effects on podocytes. Because our data showed that podocyte apoptosis increased in both DKD mice and HG-induced mouse podocytes, and MYDGF treatment attenuated this effect, we further measured the level of apoptosis-related Bcl-2-associated death promoter (BAD). 
Fig. 3 MYDGF restoration ameliorates albuminuria and glomerular injury in WT and $\mathrm{KO}$ mice with DKD. (a) Schematic representation of the AAV treatment protocol for WT and $\mathrm{KO}$ mice ( $n=8$ mice per group). (b) Urinary albumin levels were measured weekly after AAVMYDGF treatment ( $n=6$ mice per group). (c) Representative PAS staining and $(\mathbf{d}, \mathbf{e})$ quantitative analyses of mesangial matrix expansion and glomerular volume in glomeruli ( $n=4$ mice per group). Scale bar, $20 \mu \mathrm{m}$; original magnification $\times 400$. (f) Western blots and quantification analyses of fibronectin in glomerular lysates ( $n=3$ mice per group). (g-i) mRNA levels of fibronectin, Tgf$\beta$ and collagen IV in glomerular lysates were determined using RT-PCR ( $n=6$ mice per group). mice is relative to that of the control mice. Data are shown as the mean \pm SEM. One-way ANOVA followed by Tukey's post hoc test for multiple comparisons was used for groups of three or more. $* p<0.05$, $* * p<0.01$ vs WT-GFP group; ${ }^{\dagger} p<0.01$ vs KO-GFP group. FN, fibronectin; Vol., volume The mRNA expression of the
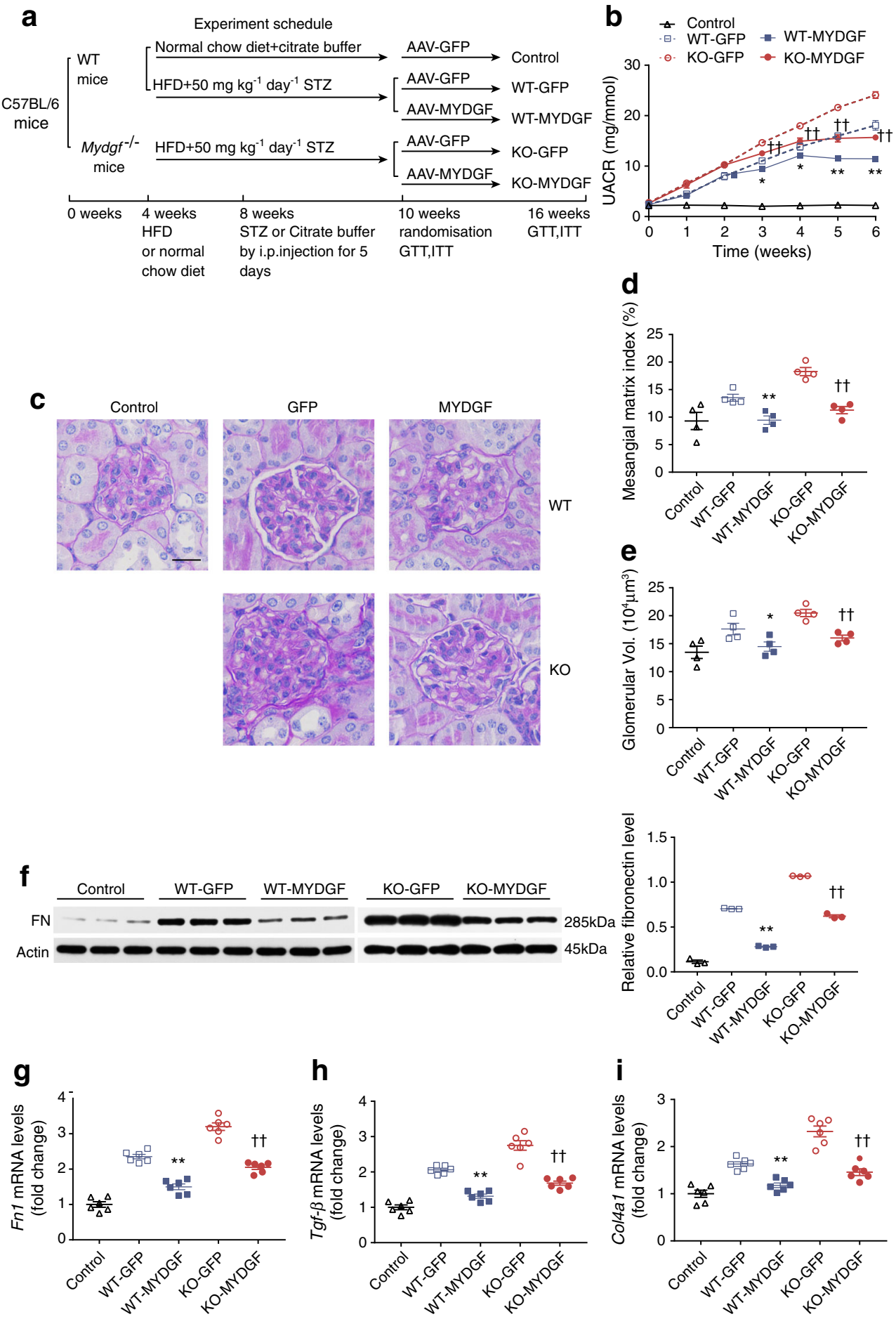

Expressed as a member of the $B c l 2$ gene family, BAD had been shown to inhibit apoptosis in its phosphorylated form [32]. Consistent with previous studies [33, 34], in our in vitro study, podocytes treated with $\mathrm{HG}$ exhibited a decrease in $\mathrm{p}-\mathrm{BAD}$, and rMYDGF treatment partially reversed this defect (Fig. 6a, b). It is well known that p-BAD can be regulated by several kinases, such as Akt, protein kinase A (PKA), p90 ribosomal S6 kinase (RSK) and p70 ribosomal S6 kinase (S6K) [35-37].
Our study found that rMYDGF treatment obviously increased p-Akt but did not alter the expression of p-PKA and p-RSK (Fig. 6a, c-f). S6K is also a downstream target of Akt and can phosphorylate BAD [38]; p-S6K declined in HG groups, but rMYDGF treatment did not change the decline (Fig. 6a, g). Consistently, our in vivo experiment showed that p-Akt and p-BAD declined in the DKD groups, and AAV-MYDGF treatment improved the decline (Fig. 6h-k). 
Fig. 4 MYDGF restoration attenuates podocyte injury and apoptosis in WT and KO mice with DKD. (a) Representative immunofluorescence images of nephrin in glomeruli. Scale bar, $20 \mu \mathrm{m}$; original magnification $\times 400$. (b) Representative immunofluorescence images of podocin in glomeruli. Scale bar, $20 \mu \mathrm{m}$; original magnification $\times 400$. (c) Representative TEM images of glomeruli. Scale bar, $1 \mu \mathrm{m}$; original magnification $\times 5000$. (d) Representative images showing WT-1 (green) and TUNEL (red) double staining. Scale bar, $20 \mu \mathrm{m}$; original magnification $\times 400$. $(\mathbf{e}, \mathbf{f})$ Quantification analyses of mean GBM thickness and mean foot process width in (c) $(n=5$ mice per group). (g) Absolute count of the numbers of glomerular cells positive for both WT-1 and TUNEL (white arrows) as the means of 20 glomeruli in (d) $(n=$ 4 mice per group). (h) Western blots and quantification analyses of nephrin and desmin in glomerular lysates ( $n=3$ mice per group). Data are shown as the mean \pm SEM. One-way ANOVA followed by Tukey's post hoc test for multiple comparisons was used for groups of three or more. ${ }^{*} p<0.05, * * p<0.01$ vs WT-GFP group; ${ }^{\dagger \dagger} p<0.01 \mathrm{vs}$ KO-GFP group
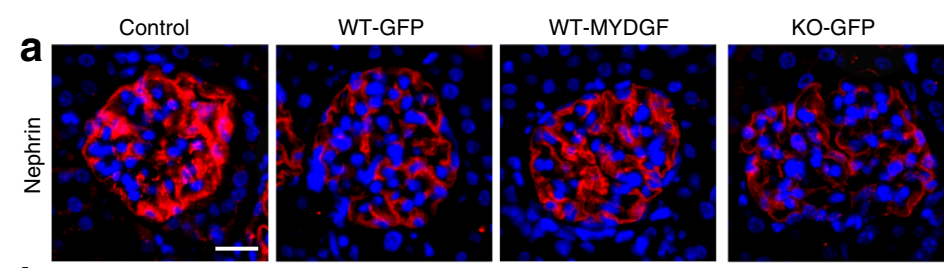

KO-MYDGF
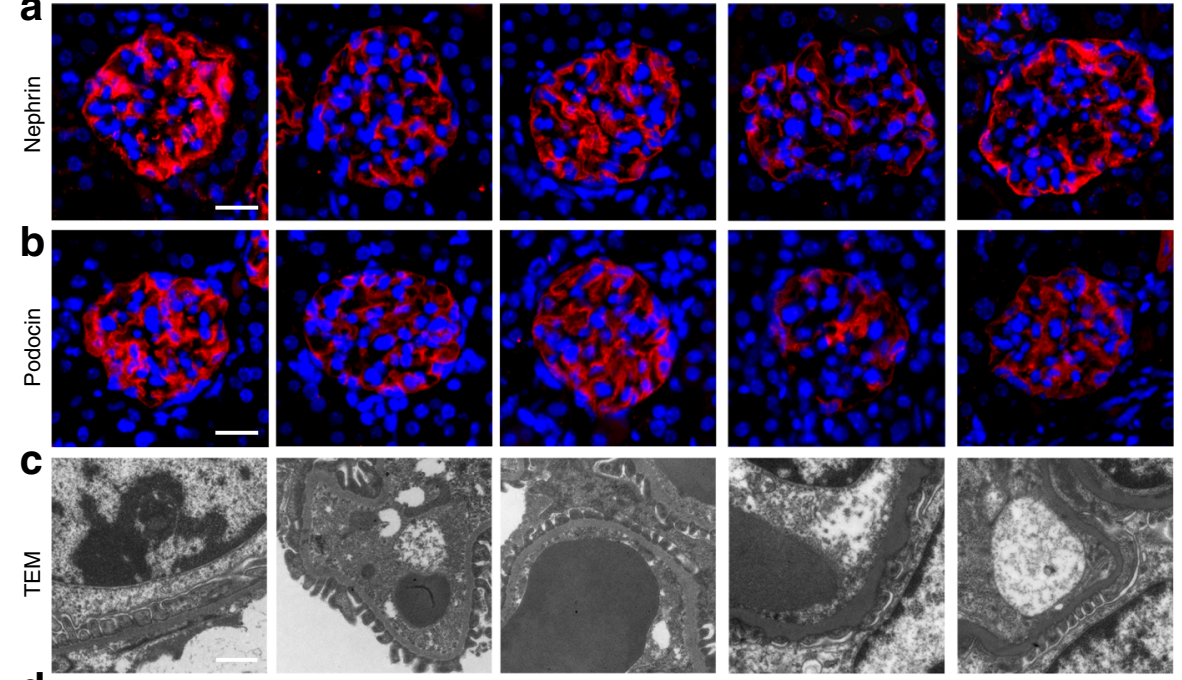

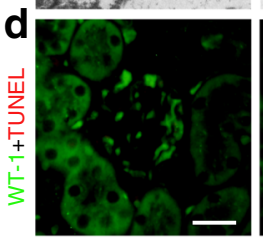

e

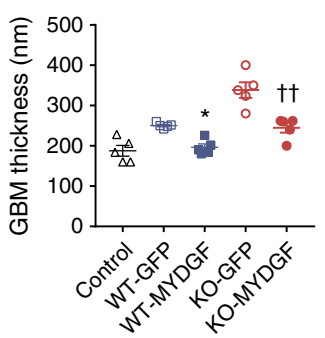

h

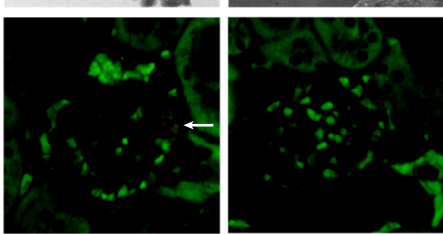

f

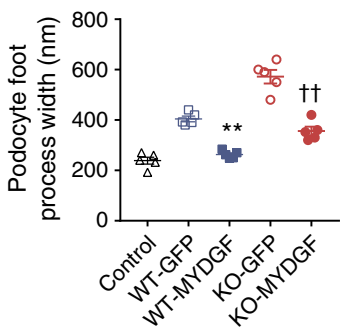

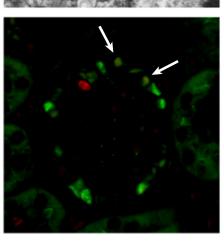

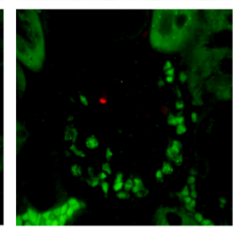

g

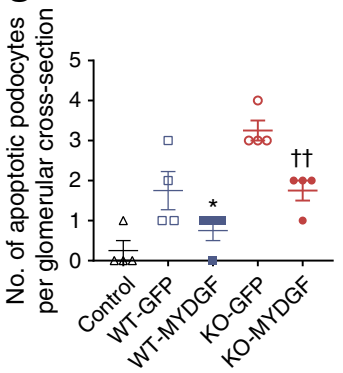

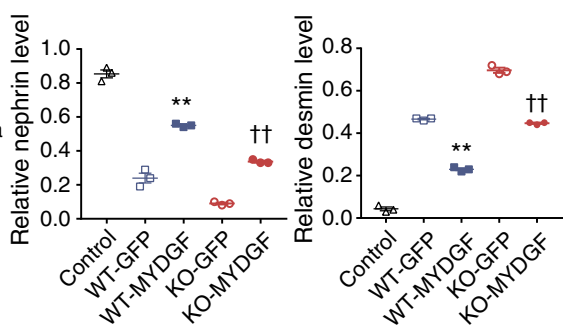

We then confirmed the involvement of the Akt/BAD pathway in the benefits of MYDGF by RNA interferencemediated gene silencing in vivo and in vitro. For siRNA treatment, the renal cortex in both kidneys of mice were injected with siAkt or siCON (Fig. 7a). After 7 days, we evaluated the silencing efficiency; as expected, a robust reduction in Akt expression was found in the kidneys, but did not occur in the liver (ESM Fig. 9a-d). After the siAkt treatment, p-BAD/ BAD in glomeruli decreased (Fig. 7b, c), and the UACR level and glomerular injury increased (Fig. $7 \mathrm{~d}-\mathrm{j}$ ). In cultured podocytes in vitro, siAkt effectively silenced Akt expression in podocytes (ESM Fig. 9e-g). As a result, Akt knockdown partially abated the rMYDGF-mediated protection of podocytes (Fig. $5 \mathrm{a}-\mathrm{f}$ ). Taken together, these data substantiate that MYDGF promotes podocyte survival partially through the Akt/BAD-dependent pathway.

\section{Bone marrow-derived MYDGF prevents albuminuria, podocyte injury and glucose metabolism in DKD mice}

Based on the fact that MYDGF is a paracrine-acting protein derived from BMDCs [17], bone marrow chimeric mice were generated to specifically address the importance of BMCderived MYDGF for DKD (Fig. 8a). Six weeks after BMC 

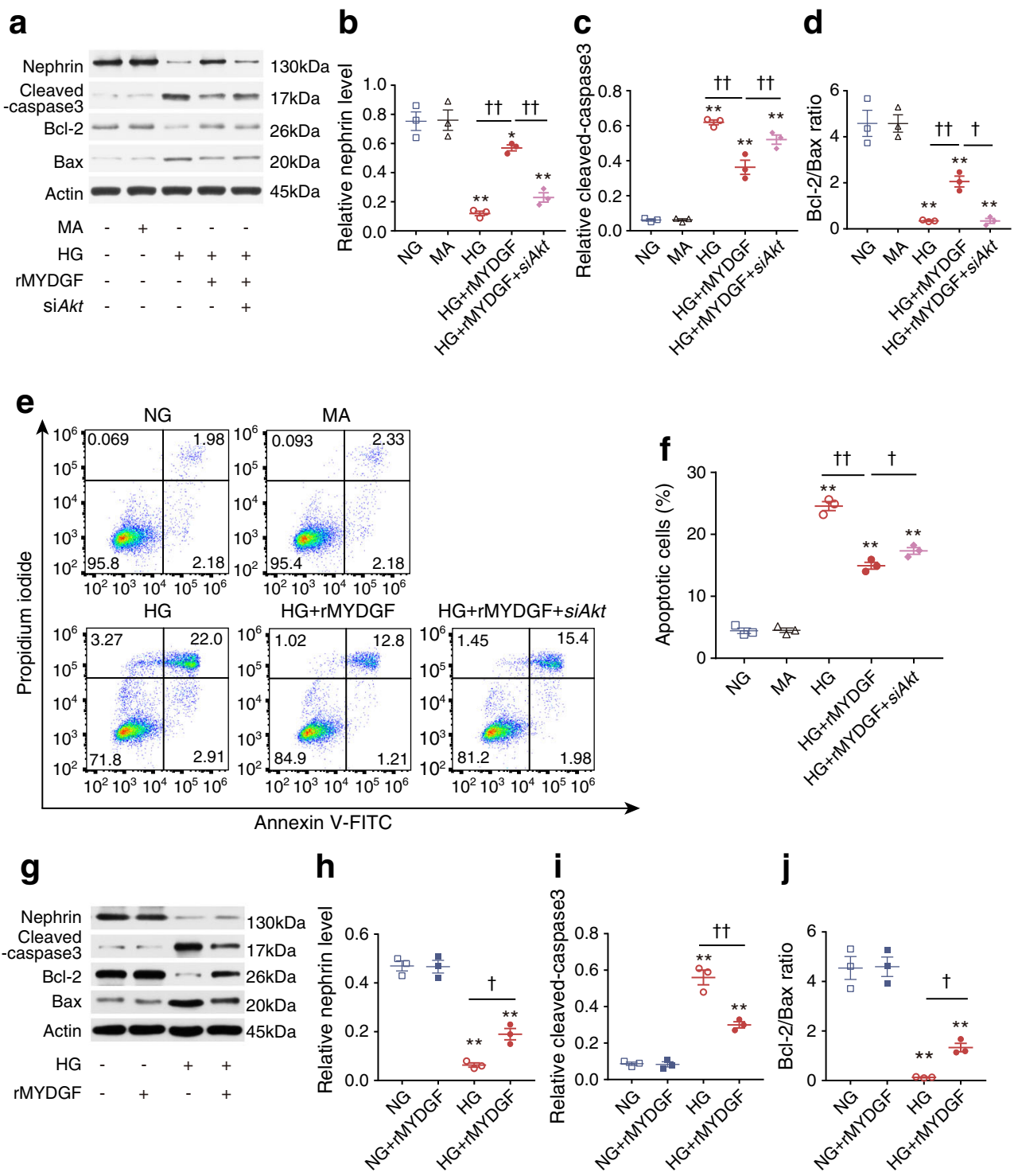

Fig. 5 MYDGF improves HG-induced podocyte injury and apoptosis in vitro and ex vivo. (a-f) Conditionally immortalised mouse podocytes (MPC5) treated with NG $(5.5 \mathrm{mmol} / 1$ glucose $), \mathrm{HG}$ (25 mmol/1 glucose) or mannitol $(5.5 \mathrm{mmol} / 1$ glucose $+19.5 \mathrm{mmol} / 1 \mathrm{D}-$ mannitol$)$ for $24 \mathrm{~h}$. Transfected with siAkt or not, HG-treated podocytes were exposed to $100 \mathrm{ng} / \mathrm{ml} \mathrm{rMYDGF}$ for another $24 \mathrm{~h}$. In addition, the NG group, MA group and HG group without rMYDGF treatment were processed in parallel without changing culture medium. (a-d) Representative western blots and quantification analyses of nephrin, cleaved caspase-3, Bax and Bcl-2 proteins in podocytes after incubation. (e, f) Apoptosis rates of

podocytes were measured by annexin $\mathrm{V}$-propidium iodide staining and flow cytometry analysis. $(\mathbf{g}-\mathbf{j})$ Isolated glomeruli were incubated with $\mathrm{NG}(5.5 \mathrm{mmol} / \mathrm{l})$ or $\mathrm{HG}(25 \mathrm{mmol} / \mathrm{l})$ for $96 \mathrm{~h}$ and then exposed to $100 \mathrm{ng} / \mathrm{ml} \mathrm{rMYDGF}$ or not for another $24 \mathrm{~h}$ without changing culture medium. Representative western blots and quantification analyses of nephrin, cleaved caspase-3, Bax and Bcl-2 proteins in glomeruli after incubation. Data are represented as the mean $\pm \mathrm{SEM}$ of three independent experiments. One-way ANOVA followed by Tukey's post hoc test for multiple comparisons was used for groups of three or more. $* p<0.05$, $* * p<0.01$ vs NG group; ${ }^{\dagger} p<0.05,{ }^{\dagger \dagger} p<0.01$ vs HG+rMYDGF group

transplantation, the plasma MYDGF levels were measured to determine chimerism. No MYDGF was detectable in the plasma of the $\mathrm{KO}$ recipient mice transplanted with KO-BMCs, and the plasma MYDGF level increased in the $\mathrm{KO}$ recipient mice transplanted with WT-BMCs (Fig. 8b). However, the plasma level of MYDGF was obviously lower in the WT recipient mice receiving $\mathrm{KO}-\mathrm{BMCs}$ than the $\mathrm{WT}$ recipient mice receiving WT-BMCs (Fig. 8b). After $\mathrm{HFD} / \mathrm{STZ}$ induction, transplantation of WT-BMCs into KO recipient mice markedly ameliorated albuminuria and glomerular injury concomitant with improvement in glucose metabolism (Fig. 8c-i, ESM Fig. 10). In contrast, transplantation of KO-BMCs into WT recipient mice worsened albuminuria and glomerular injury accompanied by impaired glucose metabolism (Fig. 8c-i, ESM Fig. 10). These results reveal that MYDGF produced by BMCs is sufficient and required for renoprotection in DKD mice. 
Fig. 6 MYDGF activates Akt/ BAD signalling in vivo and in vitro. (a) Representative western blot analyses for $\mathrm{p}-\mathrm{BAD}$ (S136), BAD, p-Akt (S473), pAkt (T308), Akt, p-PKA (T197), PKA, p-RSK (S381), RSK, pS6K (T389) and S6K in podocytes. (b-g) Bar graphs show the average ratios of phosphorylated protein signal vs total protein signal. Data are shown as the mean \pm SEM of three independent experiments. One-way ANOVA followed by Tukey's post hoc test for multiple comparisons was used for groups of three or more. $* * p<0.01 \mathrm{vs}$ NG group; ${ }^{\dagger \dagger} p<0.01$ vs HG group. (h) Representative western blots of p-BAD (S136), BAD, pAkt (S473), p-Akt (T308) and Akt in glomerular lysates. (i-k) Quantitative analysis of (h) $(n=3$ mice per group). Data are shown as the mean \pm SEM. One-way ANOVA followed by Tukey's post hoc test for multiple comparisons was used for groups of three or more. $* * p<0.01 \mathrm{vs}$ WT-GFP group; ${ }^{\dagger \dagger} p<0.01$ vs KO-GFP group. NS, not significant

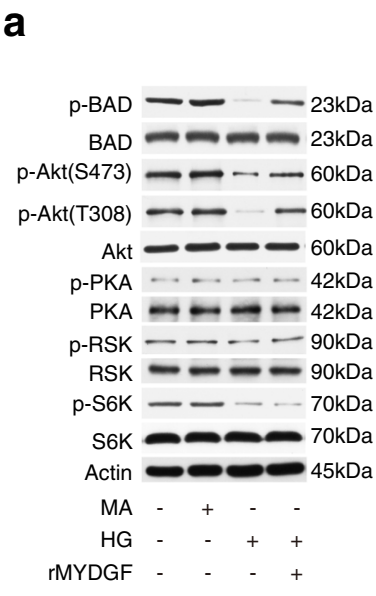

b
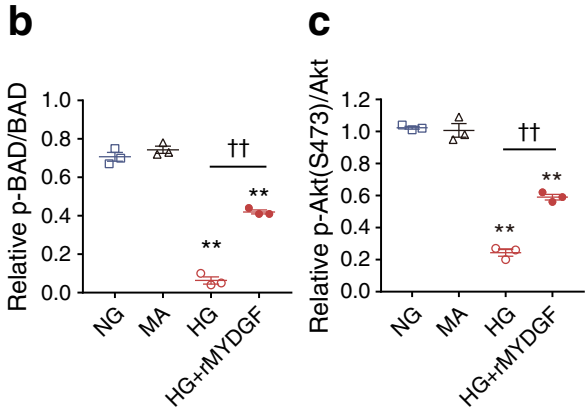

d
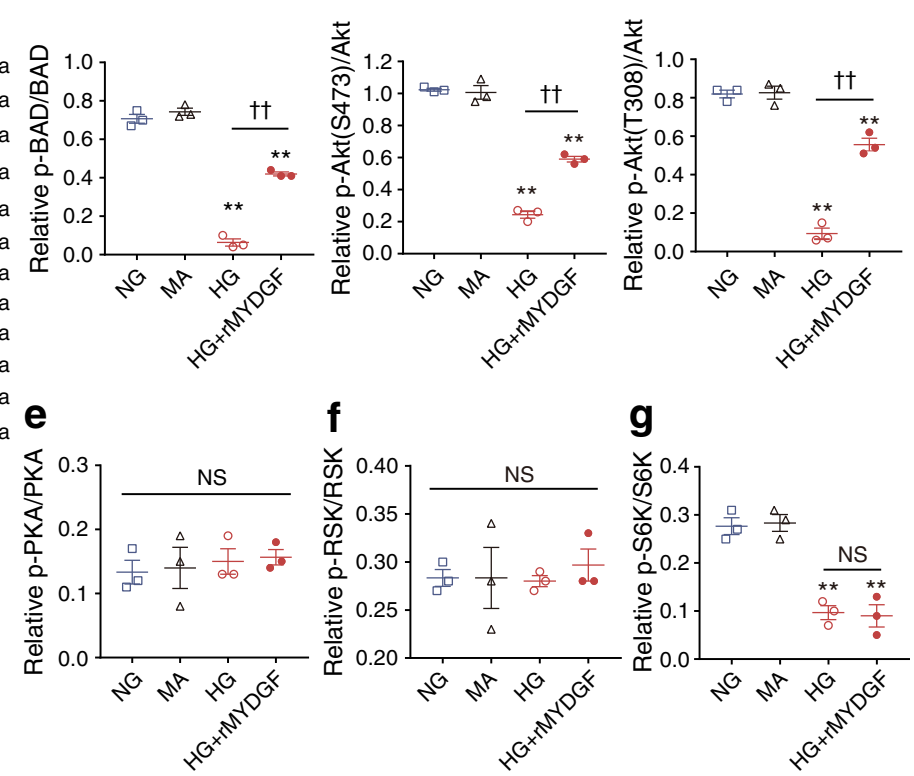
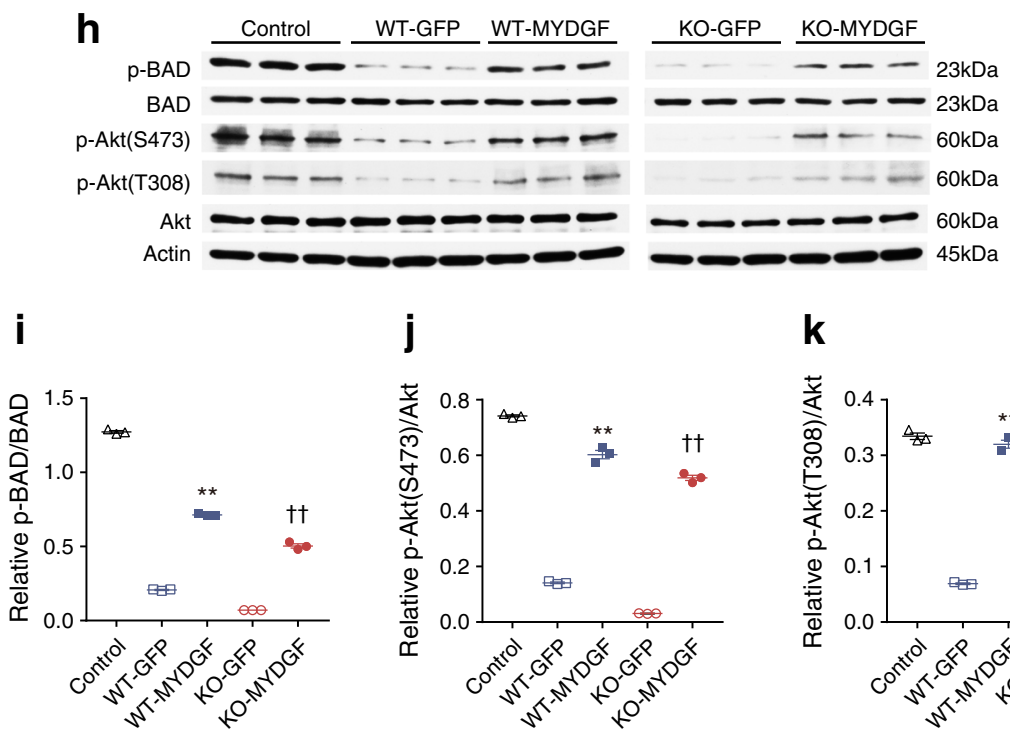

j

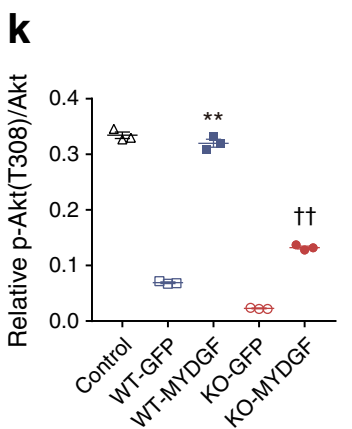

\section{Discussion}

The major findings of this study are that: (1) MYDGF ameliorates albuminuria and glomerular injury in DKD; (2) MYDGF preserves slit diaphragm protein expression and protects against podocyte apoptosis both in vivo and in vitro; and (3) the molecular mechanisms underlying these beneficial effects of MYDGF involve the activation of the Akt/BAD signalling pathway. To the best of our knowledge, our data show for the first time that MYDGF reverses damage to podocytes from diabetes and prevents the progression of DKD.

The main pathological features of DKD include glomerular hypertrophy, glomerular and tubular cell injury, mesangial matrix expansion and GBM thickening, contributing to glomerulosclerosis, tubulointerstitial degeneration and glomerular capillary dysfunction, which results in increasing albuminuria and a gradual decrease in kidney function [39, 40]. In the present study, both loss- and gain-of-function strategies indicated that MYDGF significantly attenuated the development of DKD. Under the STZ/HFD induction, WT mice developed severe albuminuria and presented pathologic changes characteristic of DKD, such as glomerular hypertrophy, mesangial matrix expansion and GBM thickening. Moreover, these lesions were more severe in KO-DKD mice than WT-DKD mice. Conversely, overexpression of exogenous $M y d g f$ gene remarkably ameliorated these lesions in both WT-DKD and KO-DKD mice. Overall, these data indicate an unequivocal role for MYDGF in preventing albuminuria and glomerular injury in DKD. 


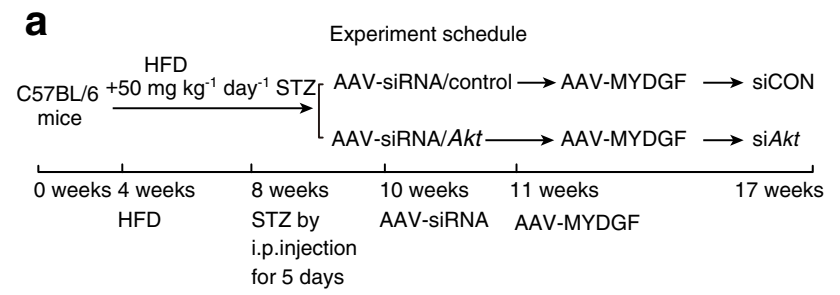

b
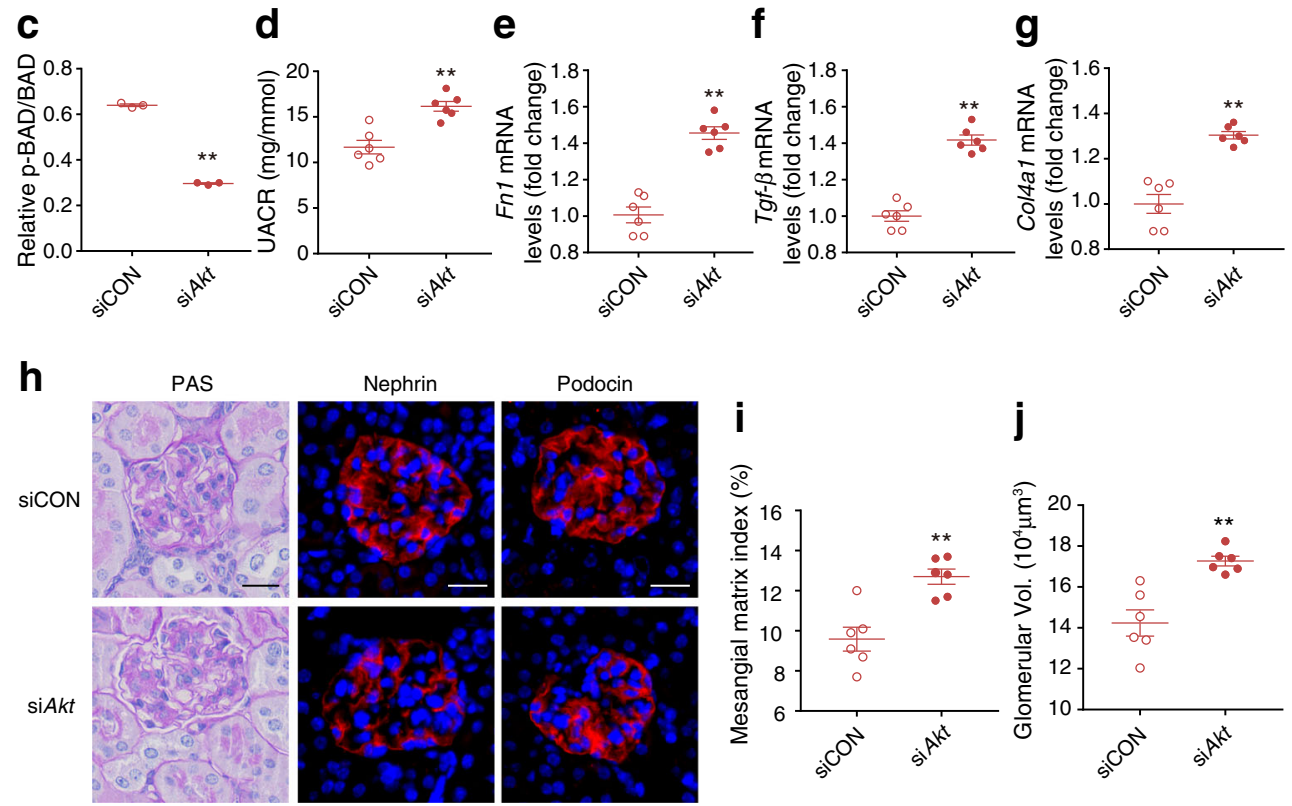

Fig. $7 A k t$-specific siRNA vitiates the positive effects of MYDGF on podocytes in vivo. (a) The experiment schedule of the HFD/STZ-induced WT-DKD mice receiving an injection of siAkt or $\operatorname{siCON}(n=10$ mice per group). (b) Western blots and (c) quantification analyses of p-BAD and $\mathrm{BAD}$ in glomerular lysates at the end of study ( $n=3$ mice per group). (d) Urinary albumin levels were measured at the termination of the study ( $n=6$ mice each group). (e-g) mRNA levels of fibronectin, $\operatorname{Tg} f-\beta$ and collagen IV in glomerular lysates were determined using RT-PCR $(n=6$

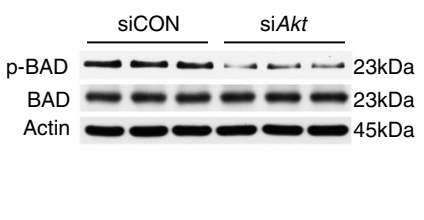

mice each group). The mRNA expression of the siAkt mice is relative to that of the siCON mice. (h) Representative PAS staining and immunofluorescence images of nephrin and podocin in glomeruli. Scale bar, $20 \mu \mathrm{m}$; original magnification $\times 400$. (i, j) Quantitative analyses of mesangial matrix expansion and glomerular volume in glomeruli $(n=6$ mice per group). Data are shown as the mean \pm SEM. Student's $t$ test was employed for comparisons between two groups. $* * p<0.01 \mathrm{vs} \mathrm{siCON}$.; Vol. volume
Podocytes play a crucial role in the regulation of the glomerular filtration barrier. Nephrin and podocin are key components of the slit diaphragm that function as the major size- and charge-selective barrier to protein leakage [41]. Therefore, podocyte injury, including podocyte loss and the disruption of slit diaphragm proteins, plays an important role in the pathogenesis of DKD $[42,43]$. In our in vivo experiment, podocin and nephrin expression decreased and desmin expression increased in the DKD mice, accompanied by increased podocyte apoptosis. Mydgf deletion significantly sharpened the decrease in slit diaphragm proteins and the increase in desmin expression and podocyte apoptosis. In contrast, overexpression of MYDGF markedly enhanced nephrin and podocin expression and decreased desmin expression and podocyte apoptosis in both WT-DKD and KO-DKD mice. Consistently, in both in vitro and ex vivo experiments, exposure of podocytes and glomeruli to HG led to the disruption of slit diaphragm protein and podocyte apoptosis, and rMYDGF treatment significantly ameliorated these effects.
Taken together, our findings establish that MYDGF treatment prevents podocyte injury by preserving slit diaphragm protein expression and decreasing podocyte apoptosis in DKD.

Next, we explored the signalling pathways that may explain the renoprotection of MYDGF in DKD. It is well known that the phosphoinositide 3-kinase (PI3K)/Akt signalling pathway plays an important role in protecting against podocyte apoptosis and improving proteinuria in DKD [44, 45]. Specifically, the activation of the PI3K/Akt signalling pathway enhances the phosphorylation of $\mathrm{BAD}$, thereby upregulating downstream $\mathrm{Bcl}-2$ and $\mathrm{Bcl}-\mathrm{xl}$ and decreasing Bax and cleaved caspase- 3 , followed by an increase in the expression of the podocyte function protein nephrin $[34,46]$. In the present study, we observed significant decreases in the phosphorylation levels of Akt and BAD in both DKD mice and HG-induced podocytes. Moreover, this inactivation of the Akt/BAD signalling pathway was partially abrogated after MYDGF treatment. Importantly, the protective effect of MYDGF is abolished by a blockade of Akt activation. 
Fig. 8 Bone marrow-derived MYDGF prevents albuminuria and podocyte injury in DKD mice. (a) The experiment schedule of WT and KO mice receiving BMCs from $\mathrm{WT}$ or $\mathrm{KO}$ mice ( $n=8$ mice per group). (b) Six weeks after bone marrow transplantation, plasma MYDGF levels were measured ( $n=8$ mice per group). (c) Urinary albumin levels were measured at the termination of the study $(n=8$ mice each group). (d-f) mRNA levels of fibronectin, $\operatorname{Tgf}-\beta$ and collagen IV in glomerular lysates were analysed by RT-PCR ( $n=6$ mice per group). The mRNA expression of the mice is relative to that of the WT $\rightarrow$ WT mice. (g) Representative PAS staining and immunofluorescence images of nephrin and podocin in glomeruli. Scale bar, $20 \mu \mathrm{m}$; original magnification $\times 400$. $(\mathbf{h}, \mathbf{i})$ Quantitative analyses of mesangial matrix expansion and glomerular volume in glomeruli ( $n=3$ mice per group). Data are shown as the mean \pm SEM. Oneway ANOVA followed by

Tukey's post hoc test for multiple comparisons was used for groups of three or more. $* * p<0.01$ vs $\mathrm{WT} \rightarrow \mathrm{WT}$ group; ${ }^{\dagger \dagger} p<0.01$ vs $\mathrm{WT} \rightarrow \mathrm{KO}$ group. Vol., volume a

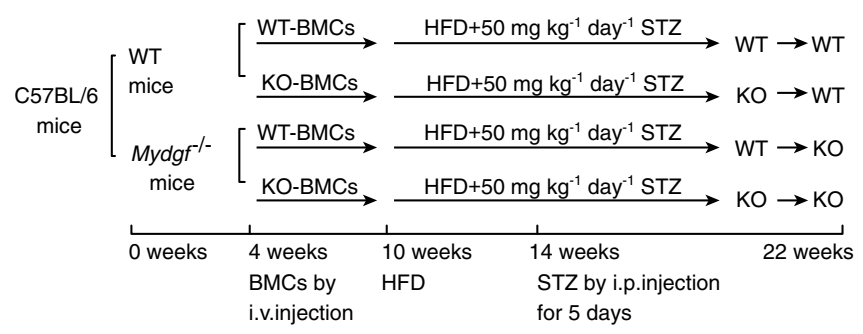

b
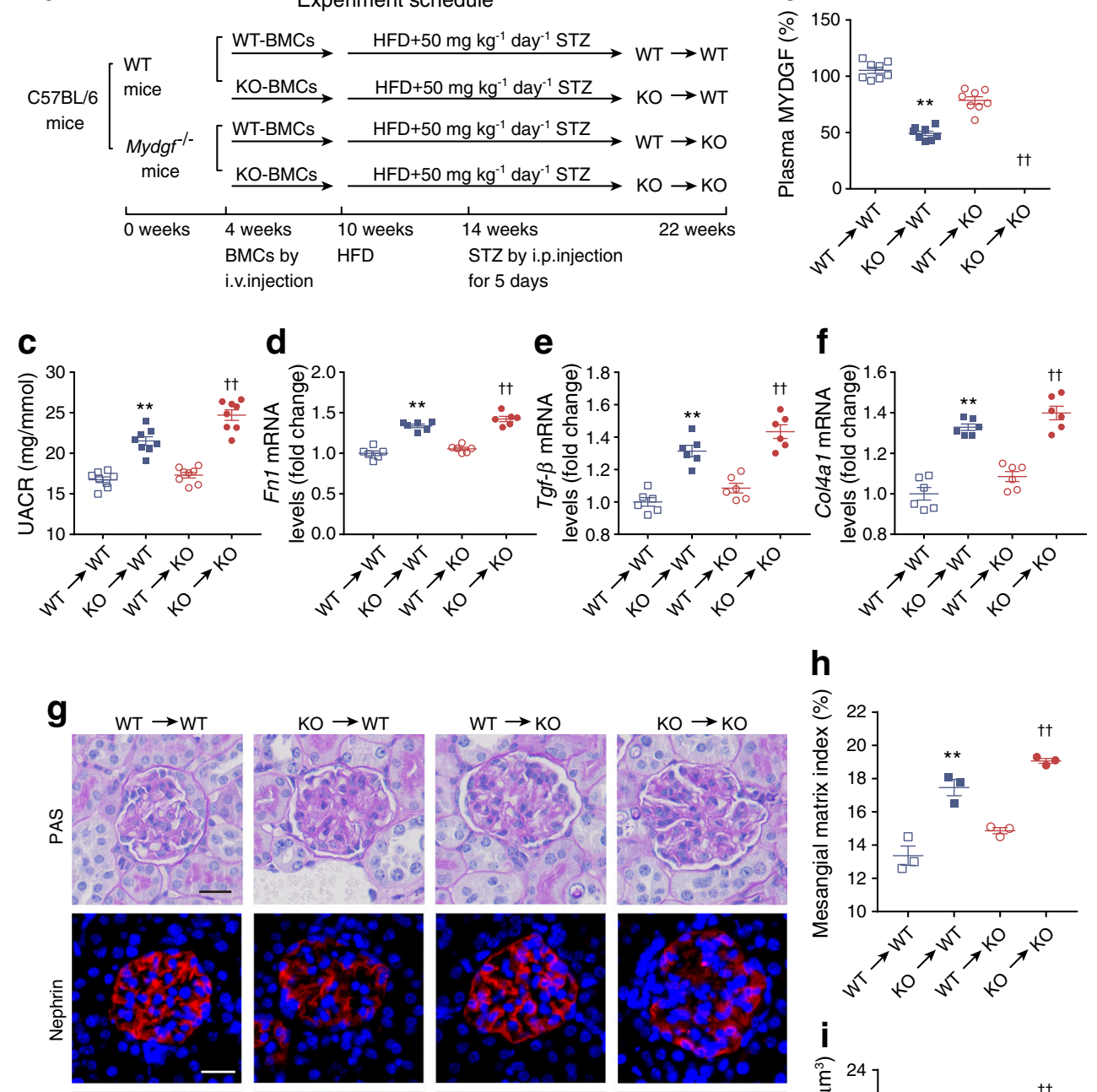

$\mathbf{h}$
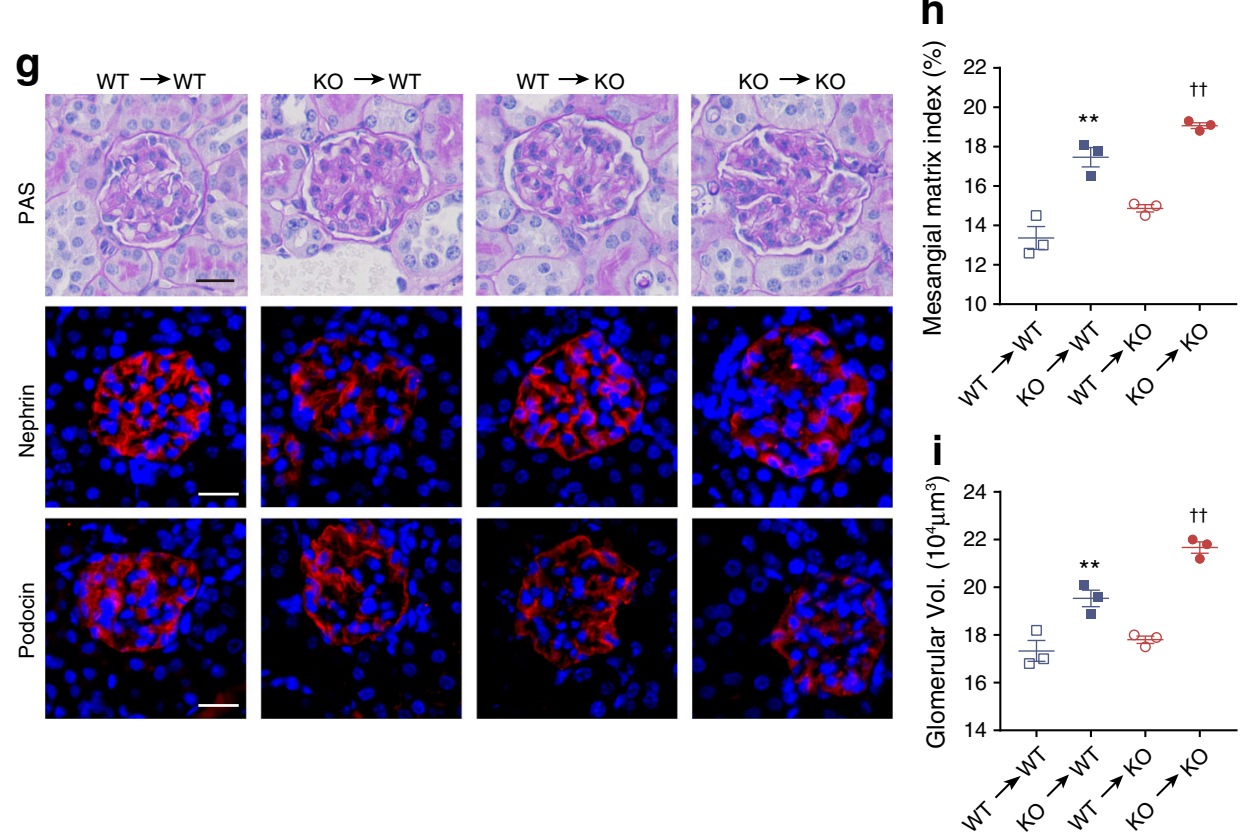

Cumulatively, we conclude that the activation of the Akt/ BAD cascade is involved in the MYDGF-mediated beneficial effects.

Herein, we also find that MYDGF improves glucose and lipid metabolism in DKD, and these positive effects may be of partial benefit for the improvement of DKD. Of note, evidence in animals and humans has shown that GLP-1 can attenuate DKD $[47,48]$. Our previous study has revealed that MYDGF stimulates GLP-1 production from intestinal L-cells in diabetic mice [18]. The increased GLP-1 may partially contribute to the improvement in DKD in the present study.

This study has some limitations. First, MYDGF is a secreted protein [49], but we have not identified the receptor(s) mediating its effects. Second, we found that MYDGF treatment improves metabolism in DKD mice, and the mechanisms need to be further investigated in other tissues, such as adipose, pancreas and liver tissues. Third, many other renal cells, such as glomerular mesangial cells, glomerular endothelial cells and proximal tubule epithelial cells, are known to be involved in the progression of DKD. However, we did not further explore the effect of MYDGF on the other renal cells. Thus, some additional studies are needed.

In summary, the data suggest a critical role for MYDGF in preventing the development of DKD by protecting podocytes from injury and improving podocyte function through the activation of the Akt/BAD pathway. The identification of 
MYDGF opens up a potential new therapeutic strategy for treating DKD and stimulates further research into the role of MYDGF in other disease states.

Data availability All data generated or analysed during this study are included in this published article (and its supplementary information files).

Funding This work was supported by grants from the National Natural Science Foundation of China (NSFC 81870573, 81370896, 81570730), National Key Research and Development Program of China (2016YFC1305601) and Research Project of Health Commission of Hubei Province (WJ2017H0031).

Authors' relationships and activities The authors declare that there are no relationships or activities that might bias, or be perceived to bias, their work.

Contribution statement MJH, YXL, LW, BG, LX, JD, JJZ and ML were responsible for data acquisition and interpretation. GDX, WM, BZ, YD, BYM and LMZ designed the study and drafted the article. LWX contributed to the conception and design of the study and analysis of data. All authors contributed to its revision and approved the manuscript for submission. GDX is the guarantor and, as such, is responsible for final approval of the version to be published.

\section{References}

1. Brosius FC, Tuttle KR, Kretzler M (2016) JAK inhibition in the treatment of diabetic kidney disease. Diabetologia 59(8):1624 1627. https://doi.org/10.1007/s00125-016-4021-5

2. Jefferson JA, Shankland SJ, Pichler RH (2008) Proteinuria in diabetic kidney disease: a mechanistic viewpoint. Kidney Int 74(1):22-36. https://doi.org/10.1038/ki.2008.128

3. Holderied A, Romoli S, Eberhard J et al (2015) Glomerular parietal epithelial cell activation induces collagen secretion and thickening of Bowman's capsule in diabetes. Lab Investig 95(3):273-282. https://doi.org/10.1038/labinvest.2014.160

4. Gruden G, Perin PC, Camussi G (2005) Insight on the pathogenesis of diabetic nephropathy from the study of podocyte and mesangial cell biology. Curr Diabetes Rev 1(1):27-40. https://doi.org/10. 2174/1573399052952622

5. Susztak K, Raff AC, Schiffer M, Bottinger EP (2006) Glucoseinduced reactive oxygen species cause apoptosis of podocytes and podocyte depletion at the onset of diabetic nephropathy. Diabetes 55(1):225-233. https://doi.org/10.2337/diabetes.55.1.225

6. Li JJ, Kwak SJ, Jung DS et al (2007) Podocyte biology in diabetic nephropathy. Kidney Int Suppl 106:S36-S42. https://doi.org/10. 1038/sj.ki.5002384

7. Zhan H, Jin J, Liang S, Zhao L, Gong J, He Q (2019) Tripterygium glycoside protects diabetic kidney disease mouse serum-induced podocyte injury by upregulating autophagy and downregulating $\beta$-arrestin-1. Histol Histopathol 34(8):943-952. https://doi.org/10. 14670/HH-18-097

8. Golle L, Gerth HU, Beul K et al (2017) Bone marrow-derived cells and their conditioned medium induce microvascular repair in uremic rats by stimulation of endogenous repair mechanisms. Sci Rep 7(1):9444. https://doi.org/10.1038/s41598-017-09883-x

9. Zhang Y, Yuen DA, Advani A et al (2012) Early-outgrowth bone marrow cells attenuate renal injury and dysfunction via an antioxidant effect in a mouse model of type 2 diabetes. Diabetes 61(8): 2114-2125. https://doi.org/10.2337/db11-1365
10. Hamza AH, Al-Bishri WM, Damiati LA, Ahmed HH (2017) Mesenchymal stem cells: a future experimental exploration for recession of diabetic nephropathy. Ren Fail 39(1):67-76. https:// doi.org/10.1080/0886022x.2016.1244080

11. Abdel Aziz MT, Wassef MA, Ahmed HH et al (2014) The role of bone marrow derived-mesenchymal stem cells in attenuation of kidney function in rats with diabetic nephropathy. Diabetol Metab Syndr 6(1):34. https://doi.org/10.1186/1758-5996-6-34

12. Castiglione RC, Maron-Gutierrez T, Barbosa CM et al (2013) Bone marrow-derived mononuclear cells promote improvement in glomerular function in rats with early diabetic nephropathy. Cell Physiol Biochem 32(3):699-718. https://doi.org/10.1159/ 000354473

13. Packham DK, Fraser IR, Kerr PG, Segal KR (2016) Allogeneic mesenchymal precursor cells (MPC) in diabetic nephropathy: a randomized, placebo-controlled, dose escalation study. E Bio Medicine 12:263-269. https://doi.org/10.1016/j.ebiom.2016.09. 011

14. Gaipov A, Taubaldiyeva Z, Askarov M et al (2019) Infusion of autologous bone marrow derived mononuclear stem cells potentially reduces urinary markers in diabetic nephropathy. J Nephrol 32(1):65-73. https://doi.org/10.1007/s40620-018-0548-5

15. Li H, Rong P, Ma X et al (2018) Paracrine effect of mesenchymal stem cell as a novel therapeutic strategy for diabetic nephropathy. Life Sci 215:113-118. https://doi.org/10.1016/j.lfs.2018.11.001

16. Nagaishi K, Mizue Y, Chikenji T et al (2016) Mesenchymal stem cell therapy ameliorates diabetic nephropathy via the paracrine effect of renal trophic factors including exosomes. Sci Rep 6: 34842. https://doi.org/10.1038/srep34842

17. Korf-Klingebiel M, Reboll MR, Klede S et al (2015) Myeloidderived growth factor (C19orf10) mediates cardiac repair following myocardial infarction. Nat Med 21(2):140-149. https://doi.org/10. 1038/nm.3778

18. Wang L, Li Y, Guo B et al (2020) Myeloid-derived growth factor promotes intestinal glucagon-like peptide-1 production in male mice with type 2 diabetes. Endocrinology 161(2). https://doi.org/ 10.1210/endocr/bqaa003

19. Du P, Fan B, Han H et al (2013) NOD2 promotes renal injury by exacerbating inflammation and podocyte insulin resistance in diabetic nephropathy. Kidney Int 84(2):265-276. https://doi.org/ 10.1038/ki.2013.113

20. Liu M, Liang K, Zhen J et al (2017) Sirt6 deficiency exacerbates podocyte injury and proteinuria through targeting Notch signaling. Nat Commun 8(1):413. https://doi.org/10.1038/s41467-01700498-4

21. Li H, Li Y, Xiang L et al (2017) GDF11 attenuates development of type 2 diabetes via improvement of islet $\beta$-cell function and survival. Diabetes 66(7):1914-1927. https://doi.org/10.2337/db17-0086

22. Mei W, Xiang G, Li Y et al (2016) GDF11 protects against endothelial injury and reduces atherosclerotic lesion formation in apolipoprotein E-null mice. Mol Ther 24(11):1926-1938. https://doi. org $/ 10.1038 / \mathrm{mt} .2016 .160$

23. Zhang J, Li Y, Li H et al (2018) GDF11 improves angiogenic function of EPCs in diabetic limb ischemia. Diabetes 67(10): 2084-2095. https://doi.org/10.2337/db17-1583

24. Gusella GL, Fedorova E, Hanss B, Marras D, Klotman ME, Klotman PE (2002) Lentiviral gene transduction of kidney. Hum Gene Ther 13(3):407-414. https://doi.org/10.1089/ 10430340252792530

25. Liu J, Feng X, Tian Y et al (2019) Knockdown of TRIM27 expression suppresses the dysfunction of mesangial cells in lupus nephritis by FoxO1 pathway. J Cell Physiol 234(7):11555-11566. https:// doi.org/10.1002/jcp. 27810

26. Bao H, Ge Y, Peng A, Gong R (2015) Fine-tuning of NFkB by glycogen synthase kinase $3 \beta$ directs the fate of glomerular 
podocytes upon injury. Kidney Int 87(6):1176-1190. https:/doi. org $/ 10.1038 / \mathrm{ki} .2014 .428$

27. van den Berg JG, van den Bergh Weerman MA, Assmann KJ, Weening JJ, Florquin S (2004) Podocyte foot process effacement is not correlated with the level of proteinuria in human glomerulopathies. Kidney Int 66(5):1901-1906. https://doi.org/10.1111/j. 1523-1755.2004.00964.x

28. Zhu B, Li Y, Mei W et al (2019) Alogliptin improves endothelial function by promoting autophagy in perivascular adipose tissue of obese mice through a GLP-1-dependent mechanism. Vasc Pharmacol 115:55-63. https://doi.org/10.1016/j.vph.2018.11.003

29. Ding Y, Jiang H, Meng B, Zhu B, Yu X, Xiang G (2019) Sweroside-mediated mTORC1 hyperactivation in bone marrow mesenchymal stem cells promotes osteogenic differentiation. $\mathrm{J}$ Cell Biochem 120(9):16025-16036. https://doi.org/10.1002/jcb. 28882

30. Alicic RZ, Rooney MT, Tuttle KR (2017) Diabetic kidney disease: challenges, progress, and possibilities. Clin J Am Soc Nephrol 12(12):2032-2045. https://doi.org/10.2215/CJN.11491116

31. Horne SJ, Vasquez JM, Guo Y et al (2018) Podocyte-specific loss of Krüppel-like factor 6 increases mitochondrial injury in diabetic kidney disease. Diabetes 67(11):2420-2433. https://doi.org/10. 2337/db17-0958

32. Yu SY, Qi R (2013) Role of BAD in podocyte apoptosis induced by puromycin aminonucleoside. Transplant Proc 45(2):569-573. https://doi.org/10.1016/j.transproceed.2012.07.160

33. Bridgewater DJ, Ho J, Sauro V, Matsell DG (2005) Insulin-like growth factors inhibit podocyte apoptosis through the PI3 kinase pathway. Kidney Int 67(4):1308-1314. https://doi.org/10.1111/j. 1523-1755.2005.00208.x

34. Kang Y, Li Y, Zhang T, Chi Y, Liu M (2019) Effects of transcription factor EB on oxidative stress and apoptosis induced by high glucose in podocytes. Int J Mol Med 44(2):447-456. https://doi. org/10.3892/ijmm.2019.4209

35. Oh J, Beckmann J, Bloch J et al (2011) Stimulation of the calciumsensing receptor stabilizes the podocyte cytoskeleton, improves cell survival, and reduces toxin-induced glomerulosclerosis. Kidney Int 80(5):483-492. https://doi.org/10.1038/ki.2011.105

36. Harada H, Becknell B, Wilm M et al (1999) Phosphorylation and inactivation of $\mathrm{BAD}$ by mitochondria-anchored protein kinase $\mathrm{A}$. Mol Cell 3(4):413-422. https://doi.org/10.1016/s1097-2765(00) 80469-4

37. Datta SR, Dudek H, Tao X et al (1997) Akt phosphorylation of BAD couples survival signals to the cell-intrinsic death machinery. Cell 91(2):231-241. https://doi.org/10.1016/s0092-8674(00) 80405-5

38. Chandrasekher G, Sailaja D (2004) Phosphatidylinositol 3-kinase (PI-3K)/Akt but not PI-3K/p70 S6 kinase signaling mediates IGF1-promoted lens epithelial cell survival. Invest Ophthalmol Vis Sci 45(10):3577-3588. https://doi.org/10.1167/iovs.04-0279
39. Mogensen CE (1971) Glomerular filtration rate and renal plasma flow in short-term and long-term juvenile diabetes mellitus. Scand J Clin Lab Invest 28(1):91-100. https://doi.org/10.3109/ 00365517109090667

40. Katsoulieris EN, Drossopoulou GI, Kotsopoulou ES, Vlahakos DV, Lianos EA, Tsilibary EC (2016) High glucose impairs insulin signaling in the glomerulus: an in vitro and ex vivo approach. PLoS One 11(7):e0158873. https://doi.org/10.1371/journal.pone. 0158873

41. Song Z, Guo Y, Zhou M, Zhang X (2014) The PI3K/p-Akt signaling pathway participates in calcitriol ameliorating podocyte injury in DN rats. Metabolism 63(10):1324-1333. https://doi.org/10. 1016/j.metabol.2014.06.013

42. Anil Kumar P, Welsh GI, Saleem MA, Menon RK (2014) Molecular and cellular events mediating glomerular podocyte dysfunction and depletion in diabetes mellitus. Front Endocrinol (Lausanne) 5:151. https://doi.org/10.3389/fendo.2014.00151

43. Barutta F, Grimaldi S, Franco I et al (2014) Deficiency of cannabinoid receptor of type 2 worsens renal functional and structural abnormalities in streptozotocin-induced diabetic mice. Kidney Int 86(5):979-990. https://doi.org/10.1038/ki.2014.165

44. Dai C, Saleem MA, Holzman LB, Mathieson P, Liu Y (2010) Hepatocyte growth factor signaling ameliorates podocyte injury and proteinuria. Kidney Int 77(11):962-973. https://doi.org/10. 1038/ki.2010.40

45. Wang XM, Yao M, Liu SX, Hao J, Liu QJ, Gao F (2014) Interplay between the Notch and PI3K/Akt pathways in high glucoseinduced podocyte apoptosis. Am J Physiol Renal Physiol 306(2): F205-F213. https://doi.org/10.1152/ajprenal.90005.2013

46. Huber TB, Hartleben B, Kim J et al (2003) Nephrin and CD2AP associate with phosphoinositide 3-OH kinase and stimulate AKTdependent signaling. Mol Cell Biol 23(14):4917-4928. https://doi. org/10.1128/mcb.23.14.4917-4928.2003

47. Fujita H, Morii T, Fujishima $\mathrm{H}$ et al (2014) The protective roles of GLP-1R signaling in diabetic nephropathy: possible mechanism and therapeutic potential. Kidney Int 85(3):579-589. https://doi. org/10.1038/ki.2013.427

48. Mann JFE, Ørsted DD, Brown-Frandsen K et al (2017) Liraglutide and renal outcomes in type 2 diabetes. N Engl J Med 377(9):839 848. https://doi.org/10.1056/NEJMoa1616011

49. Bortnov V, Annis DS, Fogerty FJ, Barretto KT, Turton KB, Mosher DF (2018) Myeloid-derived growth factor is a resident endoplasmic reticulum protein. J Biol Chem 293(34):13166-13175. https://doi. org/10.1074/jbc.ac118.002052

Publisher's note Springer Nature remains neutral with regard to jurisdictional claims in published maps and institutional affiliations. 


\section{Affiliations}

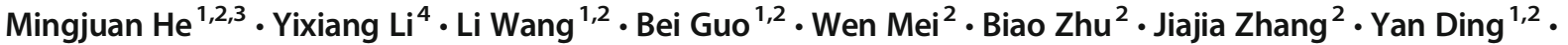
Biying Meng ${ }^{1,2} \cdot$ Liming Zhang $^{1,2} \cdot$ Lin Xiang $^{2} \cdot$ Jing Dong ${ }^{2} \cdot$ Min Liu $^{2} \cdot$ Lingwei Xiang $^{5} \cdot$ Guangda Xiang $^{1,2}$ (D)

1 The First School of Clinical Medicine, Southern Medical University, Guangzhou, Guangdong Province, China

2 Department of Endocrinology, General Hospital of Central Theater Command, Wuluo Road 627, Wuhan 430070, Hubei Province, China
3 Department of Endocrinology, Wuhan Fourth Hospital, Puai Hospital, Tongii Medical College, Huazhong University of Science and Technology, Wuhan, Hubei Province, China

4 Department of Hematology and Medical Oncology, School of Medicine, Emory University, Atlanta, GA, USA

5 ICF, 2635 Century Pkwy NE Unit 1000, Atlanta, GA 30345, USA 